Recepción: 26 / 03 / 2018

Aceptación: 15/ 05 / 2018

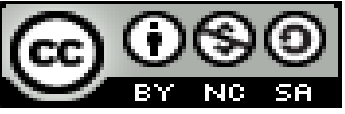

Ciencias de la Salud

Publicación: 01 / 08 / 2018

Artículo de investigación

\title{
Recidiva de pancreatitis aguda en paciente embarazada
}

Acute pancreatitis recurrence in a pregnant patient

\section{Recorrência aguda de pancreatite em paciente grávida}

\author{
Daniel Álvarez-Guerrero ${ }^{\mathrm{I}}$ \\ danyalvarezg@hotmail.com \\ Stefanía Quiñonez-Vivas II \\ milizz1548@ hotmail.com \\ Galo J. Vinueza-Aguay III \\ galojvinueza@uta.edu.ec
}

Correspondencia: danyalvarezg@hotmail.com

${ }^{\text {I } M e ́ d i c o, ~ U n i v e r s i d a d ~ T e ́ c n i c a ~ d e ~ A m b a t o, ~ A m b a t o, ~ E c u a d o r ~}$

II Médico, Universidad Técnica de Ambato, Ambato, Ecuador.

III Diploma Superior en Docencia en Ciencias de la Salud, Especialista en Medicina de Emergencias y Desastres, Doctor en Medicina y Cirugía, Universidad Técnica de Ambato, Ambato, Ecuador. 


\section{Resumen}

La pancreatitis aguda (PA) es una de las principales patologías digestivas que cursan con hospitalización cuya evolución depende de su etiología. En edad gestacional es una entidad rara presentada en el $0,03-0,09 \%$ con riesgo fetal en el $60 \%$ de los casos. Se presenta el caso de una paciente femenina de 21 años de edad con edad gestacional de 26 semanas según FUM y antecedentes de pancreatitis aguda de origen biliar hace 2 semanas con resolución por tratamiento médico conservador, presentando 3 días después dolor abdominal incapacitante, náuseas, vómito de contenido bilioso, exámenes de laboratorio con valores alarmantes de amilasa, lipasa y enzimas hepáticas, llegando al diagnóstico de pancreatitis aguda recidivante secundaria a colelitiasis confirmado por ecografía. Ingresa a unidad de cuidados intensivos para monitorización continua. Se administra terapia de mantenimiento y se decide tratamiento quirúrgico. Se toma en cuenta este caso debido a la baja prevalencia que tiene la pancreatitis aguda dentro de las complicaciones de las pacientes gravídicas. La litiasis biliar que es la principal causa de pancreatitis en nuestro medio, aumenta en pacientes embarazadas debido a un incremento notable de la saturación de colesterol y una disminución de la contracción de la vesícula en respuesta a la ingesta de alimentos cotidianos. En base a este caso clínico se pretende delimitar la causa que desencadenó, el episodio inflamatorio del páncreas y los factores predisponentes para la recidiva del mismo en pacientes gestantes con características similares.

Palabras clave: pancreatitis aguda; recidiva; embarazo; tratamiento quirúrgico.

\section{Abstract}

Acute pancreatitis (AP) is one of the main digestive pathologies that occur with hospitalization whose evolution depends on its etiology. In gestational age it is a rare entity presented in 0.03 $0.09 \%$ with fetal risk in $60 \%$ of cases. We present the case of a female patient of 21 years of age with a gestational age of 26 weeks according to FUM and a history of acute pancreatitis of biliary origin 2 weeks ago with resolution by conservative medical treatment, presenting 3 days later with disabling abdominal pain, nausea, vomiting. Of bilious content, laboratory tests with alarming values of amylase, lipase and liver enzymes, reaching the diagnosis of recurrent acute pancreatitis secondary to cholelithiasis confirmed by ultrasound. Enter the intensive care unit for continuous monitoring. Maintenance therapy is administered and surgical treatment is decided. 
This case is taken into account due to the low prevalence of acute pancreatitis within the complications of gravid patients. The biliary lithiasis that is the main cause of pancreatitis in our environment increases in pregnant patients due to a remarkable increase in cholesterol saturation and a decrease in the contraction of the gallbladder in response to the intake of daily foods. Based on this clinical case, it is intended to delimit the cause that triggered the inflammatory episode of the pancreas and the predisposing factors for its recurrence in pregnant patients with similar characteristics.

Keywords: acute pancreatitis; recurrence pregnancy; surgical treatment.

\section{Resumo}

A pancreatite aguda (PA) é uma das principais patologias digestivas que ocorrem na hospitalização, cuja evolução depende de sua etiologia. Em idade gestacional, é uma entidade rara apresentada em 0,03 - 0,09\% com risco fetal em $60 \%$ dos casos. O caso de uma paciente de 21 anos com a idade gestacional de 26 semanas apresentados por LMP e história de pancreatite gallstone aguda dois semanas atrás com resolução de tratamento médico conservador, apresentando três dias após a desativação dor abdominal, náuseas, vómitos, testes de laboratório biliosas com níveis alarmantes de amilase, lipase e teor de enzima hepática, atingindo o diagnóstico de pancreatite aguda secundária a colelitíase recorrente confirmada por ultra-som. Entre na unidade de terapia intensiva para monitoramento contínuo. A terapia de manutenção é administrada e o tratamento cirúrgico é decidido. Este caso é levado em conta devido à baixa prevalência de pancreatite aguda nas complicações de pacientes grávidas. Doença do cálculo biliar é a principal causa de pancreatite em nosso ambiente, aumenta em pacientes grávidas devido a um aumento significativo na saturação de colesterol e uma diminuição contração da vesícula biliar em resposta à ingestão diária de alimentos. Com base nesse caso clínico, pretendese delimitar a causa que desencadeou o episódio inflamatório do pâncreas e os fatores predisponentes para sua recorrência em gestantes com características semelhantes.

Palavras chave: pancreatite aguda; recorrência gravidez; tratamento cirúrgico. 


\section{Introducción}

La pancreatitis aguda (PA), es la principal entidad gastrointestinal en pacientes hospitalizados. La incidencia del cuadro agudo varía dependiendo de la causa (bebidas alcohólicas, hipertrigliceridemia, cálculos vesiculares, fármacos, etc.). La incidencia anual varía de 13 a 45/100000, sin embargo en dependencia de la etiología que desencadenó la enfermedad se puede llegar a tener una recidiva siendo las principales causas (enfermedad oculta del árbol biliar, fármacos, abuso de alcohol, hipertrigliceridemia, hipercalcemia, páncreas divisum, etc.) (1); por lo tanto, se realiza esta revisión de caso en un paciente femenina de 21 años con un embarazo actualmente de 26 semanas, donde tres días después dada el alta es ingresada nuevamente a hospitalización por una recidiva de un episodio de pancreatitis aguda de 12 días de evolución.

\section{Introducción al problema}

En el Hospital General Docente Ambato, ubicado en la zona centro de la Región Interandina del Ecuador se da la presentación clínica en una paciente cursando su segundo trimestre de embarazo con una pancreatitis aguda, y neumonía adquirida en la comunidad. Como complicación, aparece un derrame pleural bilateral que disminuye notablemente la saturación de oxígeno, siendo ingresada a la Unidad de Cuidados Intensivos. En donde recibe tratamiento y posterior a 12 días es dada de alta. Surge la interrogante de si, el derrame pleural fue consecuencia directa de la neumonía o del proceso inflamatorio del páncreas.

Tres días después de dar el alta a la paciente, es ingresada nuevamente por presentar manifestaciones clínicas de un cuadro de pancreatitis aguda. En nuestro medio la principal causa de la patología mencionada es de origen biliar en el primer episodio. En caso de una recidiva no se tienen datos estadísticos, de que etiología la causa. Es por ello que se indaga en las manifestaciones clínicas, estudios de laboratorio e imagen acompañada de fuentes bibliográficas, revisión de artículos científicos y consultas a diferentes Médicos especialistas.

La importancia de este estudio radica en el aporte del análisis de caso de una paciente con pancreatitis aguda que presenta un embarazo y una posterior recidiva, se pretende delimitar la etiología, junto a los factores de iniciación en el primer episodio y los que precipitaron o favorecieron a la aparición de un segundo episodio. Además de un diagnóstico nosológico, la terapéutica utilizada en esta paciente embarazada y la afectación respiratoria. 
El abordaje multidisciplinario de los pacientes toma cada vez más protagonismo, esto incluye la valoración clínica por parte de las especialidades inmersas en este caso: ginecología, medicina interna, gastroenterología, cirugía general y la unidad de cuidados intensivos junto a los conocimientos, protocolos de manejo y la experiencia propia de cada médico. Instaurando así el tratamiento más eficaz, seguro y conveniente; evaluando la evolución al unísono de las posibles complicaciones.

\section{Metodología}

Se realiza una presentación de caso de una paciente femenina de 21 años de grupo étnico mestiza, sin antecedentes patológicos personales, de 25 semanas de embarazo que acude al servicio de Emergencia del Hospital General Docente Ambato, donde le diagnostican Pancreatitis Aguda tipo B o de origen Biliar y Atelectasia pulmonar izquierda. Posterior sufre una complic ación con un derrame pleural izquierdo, recibe tratamiento evoluciona favorablemente en la unidad de Cuidados intensivos en dicho hospital por lo que es dada de alta, luego de 12 días vuelve a ingresar con una recidiva de la pancreatitis. El estudio es de tipo observacional y descriptivo. Se analiza un caso clínico, sus manifestaciones semiológicas, estudios de laboratorio e imagen acompañada de fuentes bibliográficas, revisión de artículos científicos y consultas a diferentes Médicos especialistas y la información obtenida a acerca de la patología en distintas revisiones bibliográficas.

\section{Resultados}

El 28 de enero del 2018 a las 12:30 del mediodía, en la ciudad de Ambato una paciente femenina de 21 años de edad, soltera, de etnia mestiza, nacida y residente en Ambato, de bajo nivel socioeconómico gestante de 26,2 semanas por Fecha de última menstruación (FUM). Con antecedentes de pancreatitis aguda, atelectasia y derrame pleural izquierdo menor al 30\% hace 15 días (13 de enero de 2018), acude al servicio de emergencia del Hospital General Docente Ambato por presentar dolor abdominal de tipo continuo de característica perforante, localizado en epigastrio de gran intensidad 10/10 en la escala visual análoga (EVA) de 3 horas de evolución sin causa aparente, acompañado de nausea que llegan al vómito por 1 ocasión de contenido bilioso. Se recibe paciente en Emergencia con los siguientes signos vitales (ver tabla 1). 


\section{Tabla 1}

Signos vitales

\begin{tabular}{ll}
\hline Parámetro & Valores \\
\hline Tensión arterial & $110 / 80$ \\
\hline Temperatura & 36,0 \\
\hline Frecuencia cardiaca & 78 \\
\hline Frecuencia respiratoria & 22 \\
\hline Peso & $67 \mathrm{~kg}$ \\
\hline Escala de coma de Glasgow & $\mathrm{O} 4 \mathrm{~V} 5 \mathrm{M} 6$
\end{tabular}

Fuente: Elaboración propia.

Al examen físico paciente álgica, pálida, sudorosa, fría, abdomen gestante, duro con contracciones 1/10, movimientos fetales presentes, frecuencia cardiaca fetales 145 , altura de fondo uterino de 25 centímetros; Cefálico dorso izquierdo, dilatación entreabierto reblandecido, score mamá 0 , tirilla reactiva no realizada. Se realizan las maniobras de: Murphy positivo, Mc Burney negativo, Blumberg negativo, Psoas negativo, Valsalva negativo.

Se le administra hidratación y antiespasmódicos (butilescopolamina) a las 14:45, gestante de 26,2 semanas se valora en ginecología sin anomalías aparente en A las 17:25 del mismo día por valoración del servicio de cirugía general se decide ingreso para control del dolor con hidratación a $120 \mathrm{ml} / \mathrm{h}$, antibioterapia (ampicilina + sulbactam) más antiespasmódicos (butilescopolamina), analgésico (tramadol).A las 17:40 es traída por disposición de gerencia medica directamente a la Unidad de Cuidados Intensivos se recibe con signos vitales (ver tabla 2).

Tabla 2

Signos vitales

\begin{tabular}{ll}
\hline Parámetro & Valores \\
\hline Tensión arterial & $100 / 60$ \\
\hline Temperatura & 37,0 \\
\hline Frecuencia cardiaca & 98 \\
\hline Frecuencia respiratoria & 18 \\
\hline Peso & $67 \mathrm{~kg}$ \\
\hline Saturación de oxigeno & $95 \%$ \\
\hline Escala de coma de Glasgow & $\mathrm{O} 4 \mathrm{~V} 5 \mathrm{M} 6$ \\
\hline
\end{tabular}

Fuente: Elaboración propia. 
Al examen físico paciente despierta consciente, orientada, colaboradora, con buena saturación, dinámicamente estable sin necesidad de vasoactivos, con buena mecánica ventilatoria con apoyo de oxigeno por cánula nasal a 2 litros saturado mayor 90\%, tórax expansibilidad conservada, pulmones murmullo vesicular disminuido en campo pulmonar izquierdo en la base. Corazón rítmico no se auscultan soplos. Abdomen gestante suave depresible no doloroso, sin signos de irritación peritoneal, ruidos hidroaéreos presentes, realiza deposición, RIG presencia de líquido en claro en moderada de cantidad de mal olor, sonda vesical permeable orina coluria. Extremidades sin edemas y perfusión distal conservada. Resultados de laboratorio (ver tabla 3).

Tabla 3

Exámenes de laboratorio

\begin{tabular}{|c|c|c|c|}
\hline Fecha & 28-enero & Referencia & \\
\hline \multicolumn{4}{|c|}{ Enzimas } \\
\hline Globulinas & 3.02 & $2.30-3.50$ & $\mathrm{~g} / \mathrm{dL}$ \\
\hline AST-TGO & 57 & $0-38$ & $\mathrm{U} / 1$ \\
\hline ALT-TGP & 68 & $5-42$ & $\mathrm{U} / \mathrm{l}$ \\
\hline LDH & 160 & $240-480$ & $\mathrm{U} / \mathrm{l}$ \\
\hline Fosfatasa Alcalina & 172 & $35-104$ & $\mathrm{U} / 1$ \\
\hline Amilasa & 1755 & $28-100$ & $\mathrm{U} / \mathrm{l}$ \\
\hline Lipasa & 1267.8 & $13-60$ & $\mathrm{U} / \mathrm{l}$ \\
\hline \multicolumn{4}{|c|}{ Perfil lipídico } \\
\hline Triglicéridos & 238 & $50-200$ & $\mathrm{mg} / \mathrm{dl}$ \\
\hline Colesterol & 205 & $140-200$ & $\mathrm{mg} / \mathrm{dl}$ \\
\hline HDL-Colesterol & 33 & $45-65$ & $\mathrm{mg} / \mathrm{dl}$ \\
\hline LDL-colesterol & 134 & $0-150$ & $\mathrm{mg} / \mathrm{dl}$ \\
\hline \multicolumn{4}{|c|}{ Electrolitos } \\
\hline Sodio & 137 & $136-1450$ & $\mathrm{mEq} / \mathrm{l}$ \\
\hline Potasio & 4.20 & $3.5-5.5$ & $\mathrm{mEq} / \mathrm{l}$ \\
\hline Cloro & 100 & $96-110$ & $\mathrm{mEq} / \mathrm{l}$ \\
\hline \multicolumn{4}{|l|}{ Biometría Hemática } \\
\hline Leucocitos & 18320 & $5000-10000$ & \\
\hline
\end{tabular}

Fuente: Elaboración propia. 


\section{Discusión}

El presente reporte de caso es de una paciente femenina, de 21 años de edad, gestante de 26 semanas que presenta una recidiva de un cuadro de pancreatitis aguda de 12 días de evolución, que se reagudiza tres días posteriores al alta de la misma. Por ello se analiza este caso, en busca de la causa que desencadeno el primer episodio y los factores que precipitaron su segundo episodio. En nuestro medio las principales causas de pancreatitis aguda (PA), son la presencia de cálculos biliares o alcoholismo, debido a la ausencia de antecedentes respecto a la ingesta de bebidas alcohólicas. Se toma en cuenta que en las pacientes embarazadas se da un incremento notable de la saturación de colesterol y una disminución de la contracción de la vesícula en respuesta a la ingesta de alimentos cotidianos; apareciendo así microlitiasis y barro biliar que suelen desaparecer luego del parto en la mayoría de las mujeres; estas características aparecen en la ultrasonografía de abdomen realizada a la paciente.

Se le atribuye al primer episodio de pancreatitis aguda, a una causa de origen biliar (colelitiasis), que se la trata con terapia de mantenimiento; monitoreando y cuidando la salud materno-fetal. En la recidiva de la pancreatitis aguda, la paciente manifiesta un cuadro clínico de mayor intensidad que el primero que se acompaña de coluria y se dan elevadas cantidades de enzimas hepáticas y pancreáticas, enfocando la etiología de la recidiva de origen biliar por el aumento de las enzimas hepáticas y las disposiciones anteriormente menciona que tienen las mujeres embarazadas para la aparición de microlitiasis y barro biliar, que se dan entre el 10-30\% de las mismas (1).

En la evaluación del pronóstico en la PA, existen varios criterios clínicos y de imagen que nos ayudan a determinar el porcentaje de morbimortalidad. Se utilizaron los criterios de Ranson y Baltazar obteniendo lo siguiente:

\section{Criterios clínicos}

Criterios de Ranson - al ingreso

o Leucocitosis 18320 


\section{Criterios Imagenológicos}

- Criterios de Baltazar

o Páncreas aumentado de tamaño (1 punto)

o Rarefacción de grasa peripancréatica (2 puntos)

- Necrosis pancreática

o Sin necrosis (0 puntos)

\section{Tabla 4}

Criterios de Baltazar, CT Score

\begin{tabular}{ll}
\hline Índice de severidad tomográfica & $<\mathbf{3}$ \\
Porcentaje de complicaciones & $8 \%$ \\
Porcentaje de mortalidad & $3 \%$ \\
\hline
\end{tabular}

Fuente: Elaboración propia.

Durante su hospitalización la paciente gestante, hemodinámicamente estable, despierta, consciente, orientada, colaboradora, sin sustancias vasoactivas ni sedoanalgesia, con buena mecánica ventilatoria cánula a dos litros y controles fetales normales. Se decide su traslado a un hospital de III (tercer nivel) para realizar una Colangiopancreatografia retrógrada endoscópica (CPRE) y la valoración de un posible tratamiento quirúrgico, en ecografía abdominal se reporta la presencia de cálculos y barro biliar.

\section{Concepto}

La Pancreatitis Aguda (PA) es un proceso inflamatorio agudo que afecta a la glándula pancreática. Producido por una gran variedad de causas, tiene la característica de que con cierta frecuencia es capaz de activar una serie de sistemas inflamatorios y antiinflamatorios de efectos sistémicos que conducen a la aparición de fallo orgánico cuyas consecuencias pueden ser fatales (2). El diagnóstico de PA representa un reto en pacientes con dolor abdominal y, en caso de complicaciones eleva la morbilidad, mortalidad y costos hospitalarios (3) (4). En el $20 \%$ de los pacientes con PA adoptan un curso evolutivo grave, con aparición de sepsis, síndrome de distrés respiratorio, insuficiencia renal y fallo multiorgánico, con una tasa de mortalidad del 25\% (5). 


\section{Prevalencia e Incidencia}

La incidencia de PA de cualquier etiología, ha aumentado significativamente en las últimas décadas; aunque también su resolución ha mejorado, así como su mortalidad ha disminuido (6).Su frecuencia relativa se cifra entre 17 y 28 casos cada 100.000 habitantes, motivando del 0.15 al $1.5 \%$ de todos los ingresos hospitalarios. La mayoría de las pancreatitis se producen en pacientes con edades comprendidas entre los 50 y 75 años y sexo variable en función de la causa (7). La PA es la causa de más de 250.000 hospitalizaciones por año. El promedio de duración de la hospitalización es de cuatro días, con una mortalidad del 1\% en PA leve. Los índices de hospitalización aumentan con la edad, son $88 \%$ mayores en personas de raza negra, y son más altos en varones que en mujeres. La cifra ajustada a edades de altas hospitalarias por el diagnóstico de pancreatitis aguda aumentó a 62\% entre 1988 y 2004. Del año 2000 a 2009, la cifra aumentó $30 \%$ (1).

En el embarazo la incidencia de pancreatitis aguda (PA) es aproximadamente del 0,03 al 0,09\% de las pacientes en gravidez. Las posibles causas de PA en el embarazo son las mismas que en las pacientes no gestantes, entre las que destaca la enfermedad litiásica de la vía biliar como la causa más frecuente (67 al 100\%) (8). En el curso de la enfermedad la mortalidad materna es baja en pancreatitis no complicada, pero excede el $10 \%$ en pancreatitis complicada. La pancreatitis durante el primer trimestre se asocia a desgaste fetal y durante el tercer trimestre se asocia a trabajo de parto prematuro (9); son aspectos a tomar en cuenta mediante el curso del estado de gravidez.

\section{Etiología}

En los adultos es común una PA por litiasis o por alcoholismo, suponen más del $80 \%$ de los casos (6); por el contrario, en los niños la etiología es muy variable (10). El 10\% corresponden a etiología de origen: Idiopático, hiperlipidemias, infecciones virales, perfusión pancreática deteriorada, obstrucciones ductal, fármacos, hipercalcemia (5) (11). La incidencia de la PA aumenta con la edad, y la presentación en edades tempranas sugiere la causa hereditaria, infecciones y traumas (5) (12) (13).El riesgo de pancreatitis aguda alcohólica responde a la cantidad ingerida de alcohol (especialmente cerveza), no a la frecuencia de su consumo. Sólo 
15\% de las personas con alcoholismo crónico padecerán pancreatitis aguda (4).A continuación, se observa las diferentes etiologías clasificadas según la frecuencia de aparición: Ver tabla 4.

\section{Tabla 4}

\section{Causas de la Pancreatitis Aguda (3)}

\section{Causas Comunes}

- Litiasis vesicular (incluida la microlitiasis)

- Alcohol (alcoholismo agudo y crónico)

- Hipertrigliceridemia

- Colangiopancreatografia retrógrada endoscópica (ERCP)

- Traumatismo no penetrante del abdomen

- Estado postoperatorio (estado ulterior a operaciones abdominales y no abdominales)

- Fármacos (azatioprina, 6-mercaptopurina, sulfonamidas, estrógenos, tetraciclina, ácido valproico, fármacos contra VIH)

- Disfunción del esfínter de Oddi

Causas poco comunes

- Causas vasculares y vasculitis (estados de isquemiahipoperfusión después de operaciones del corazón)

- Conjuntivopatías y púrpura trombocitopénica trombótica

- Cáncer de páncreas

- Hipercalcemia

- Divertículo periampollar

- Páncreas dividido

- Pancreatitis hereditaria

- Fibrosis quística

- Insuficiencia renal

Causas raras

- Infecciones (parotiditis, por virus Coxsackie o citomegalovirus, echo virus y parásitos)

- Autoinmunitarias (como síndrome de Sjögren)

Causas por considerar en personas con crisis recurrentes de pancreatitis aguda sin un origen evidente 
- Enfermedad oculta de vías biliares o conductos pancreáticos, en particular microlitiasis, sedimento

- Fármacos

- Hipertrigliceridemia

- Cáncer pancreático

- Disfunción del esfínter de Oddi

- Fibrosis quística

- Causas idiopáticas

Fuente: Elaboración propia.

\section{Fisiopatología}

Existen muchas teorías por el cual se desarrolla la Pancreatitis. Una de ellas es la activación y liberación de enzimas pancreáticas hacia el intersticio, con la consiguiente autodigestión pancreática (4) (14) (15). Otros autores mencionan que la lesión del tejido pancreático es producida por dos mecanismos principales: el factor agresor (fármacos, infección o trastorno metabólico) y la activación secundaria del tripsinógeno que desencadena la respuesta inflamatoria subsecuente. Caracterizada por ser de tipo Th1, es decir, con la participación primordial de las células CD4, linfocitos B, T y monocitos. Progresivamente el daño pancreático produce isquemia microvascular y obstrucción de los acinos pancreáticos, por lo que se perpetúa el daño ocasionado por las enzimas pancreáticas (5).

La fibrosis solo ocurre cuando existe una lesión acinar recurrente con la consecuente liberación de quimiocitocinas que estimulan las células estelares. Es común que en la pancreatitis aguda grave se presenten una serie de complicaciones sistémicas, como sepsis, insuficiencia respiratoria y falla orgánica múltiple, que frecuentemente llevan a la muerte (5).

\section{Cuadro Clínico}

Dentro de los síntomas predominantes en la PA está el dolor abdominal (95-100\%) de instauración súbita, continuo, que se intensifica rápidamente hasta alcanzar su pico en tan solo unos minutos a una hora, y se localiza en epigastrio; es fijo o irradiado a uno o ambos hipocondrios, espalda, zona periumbilical y en cinturón. Al examen físico, dolor a la palpación en epigastrio siempre aparece y con frecuencia se percibe sensación de ocupación o empastamiento 
a ese nivel, incluso una masa epigástrica en algunos casos, a veces con defensa pero sin claros signos de reacción peritoneal. Los ruidos intestinales están disminuidos o están ausentes.

Puede presentarse ictericia en el $25-30 \%$ de los pacientes con PA o una rubicundez facial, relacionada esta última con la liberación de histamina y otras sustancias vasoactivas. En casos graves, el compromiso hemodinámico es evidente, con hipotensión, taquicardia o incluso shock establecido.

También pueden desarrollar un cuadro de vómitos y náuseas presentes en el 70-90\% de los casos y suele haber detención del tránsito intestinal desde el comienzo de la sintomatología. Además de distensión abdominal (60-70\%), febrícula (70-85\%), hematemesis (5\%), hipotensión (20-40\%).

El 20-35\% de los pacientes pueden desarrollar cuadros confusionales. En los casos graves también están presentes la Disnea $(<10 \%)$, taquipnea, los signos de insuficiencia cardiaca, la confusión mental o la tetania, los cuales son una expresión del compromiso multisistémico. Las equimosis abdominales de localización periumbilical (signo de Cullen) $(<5 \%)$ o en los flancos (signo de Gray-Turner) $(<5 \%)$ son muy característicos, de la pancreatitis aguda así como manifestaciones tardías de la enfermedad, generalmente en extremidades inferiores, con aspecto similar al eritema nudoso o a la paniculitis supurada (16).

\section{Diagnóstico}

Para el diagnóstico de PA se requieren al menos dos de los siguientes tres criterios (17):

- Cuadro clínico sugerente (dolor abdominal localizado en epigastrio de gran intensidad, y con irradiación a la espalda; la intensidad puede ser progresiva acompañado de náusea y vómito)

- Alteraciones bioquímicas (elevación de lipasa y/o amilasa al menos tres veces por encima del valor de referencia)

- Alteraciones estructurales tanto del páncreas como de estructuras adyacentes vistas en los estudios de imagen 
Dentro de los estudios radiológicos compatibles para el diagnóstico de PA tenemos varios. Uno de ellos es la Tomografía Axial Computarizada (TAC abdominal), muy necesario y puede utilizarse para confirmar una sospecha clínico-analítica, ya que es el método diagnóstico más fiable. La TAC realizada entre el 4 al 10 día permite el diagnóstico del 100\% de la necrosis pancreática. En la actualidad, la TAC dinámica con contraste es el patrón oro en la identificación y la cuantificación de la necrosis en las primeras 36 a 48 horas del proceso.

La Resonancia Magnética (RM) constituye un estudio de imagen muy útil en el diagnóstico; sin embargo, debemos considerar que en un gran porcentaje de los casos son pacientes graves, monitorizados y con aditamentos ferromagnéticos que contraindican este medio. El ultrasonido es el primer estudio de imagen que generalmente se realiza en estos pacientes, por su fácil accesibilidad, no es invasivo y no implica peligro de radiación para el paciente. Tradicionalmente, se consideraban como hallazgos sugestivos de pancreatitis el incremento del tamaño de la glándula pancreática y la disminución de su ecogenicidad; sin embargo, a través de los años se ha observado que estos cambios tienen una baja sensibilidad, pues hasta en 50\% de los casos de pancreatitis aguda se ha reportado una glándula de tamaño normal y solo $10 \%$ presenta hipoecogenicidad. El ultrasonido también es útil en la detección de pseudoquistes, complicación común de la pancreatitis aguda; las calcificaciones del páncreas y los cálculos intraductales pueden ser evidenciados en el ultrasonido en la pancreatitis crónica.

La colangiopancreatografia retrograda endoscópica resulta beneficiosa en los pacientes con diagnóstico de pancreatitis de origen biliar. Permite la visualización de los conductos pancreáticos y detectar dilatación, estenosis, pseudoquistes, defectos de llenado del conducto, incluyendo cálculos, tapones de moco o lodo biliar. A su vez, resalta por este medio las malformaciones estructurales de la vía pancreatobiliar en los pacientes con sospecha de pancreatitis aguda y está indicada en los casos de pancreatitis recurrente o crónica (5) (18).

\section{Pronóstico y complicaciones}

El pronóstico de los pacientes con PA depende de la sintomatología que se pueda valorar y de los diferentes criterios de gravedad donde podemos valorar el avance progresivo de esta enfermedad. La valoración clínica a pie de cama es rápida y sencilla, pero está sometida a la subjetividad del explorador y no es útil para los estudios de investigación. La mayoría de los signos y síntomas se 
presentan en un tanto por ciento reducido de enfermos o tardan en aparecer. La aplicación en el momento del ingreso de un índice elaborado con datos clínicos (criterios de gravedad: ver Tabla 5) predice adecuadamente la evolución en la PA (19); allí se puede establecer el pronóstico de los pacientes que padecen esta patología.

\section{Tabla 5}

Sistemas de Predicción de Gravedad (20)

\begin{tabular}{|c|c|c|c|c|}
\hline \multicolumn{3}{|c|}{ CLÍNICOS-ANALÍTICOS } & \multicolumn{2}{|c|}{ RADIOLÓGICOS (TC) } \\
\hline $\begin{array}{l}\text { CRISTERIOS } \\
\text { DE SRIS }\end{array}$ & $\begin{array}{l}\text { CRISTERIOS DE } \\
\text { RANSON }\end{array}$ & BISAP & $\begin{array}{l}\text { SISTEMA DE } \\
\text { BALTHAZAR }\end{array}$ & CT-SCORE \\
\hline $\begin{array}{l}\text { Frecuencia } \\
\text { cardíaca }>90 \\
\text { lpm } \\
\text { Temperatura } \\
\text { central }<36^{\circ} \mathrm{C} \\
\text { o }>38^{\circ} \mathrm{C} \\
\text { Leucocitos } \\
<4000 \quad \text { o } \\
>12000 \mathrm{~mm}^{3} \text { o } \\
>10 \% \text { formas } \\
\text { inmaduras } \\
\text { (bandas) } \\
\text { Frecuencia } \\
\text { respiratoria } \\
>20 \quad \text { rpm o } \\
\mathrm{pCO}_{2}<32 \\
\text { mmHg }\end{array}$ & 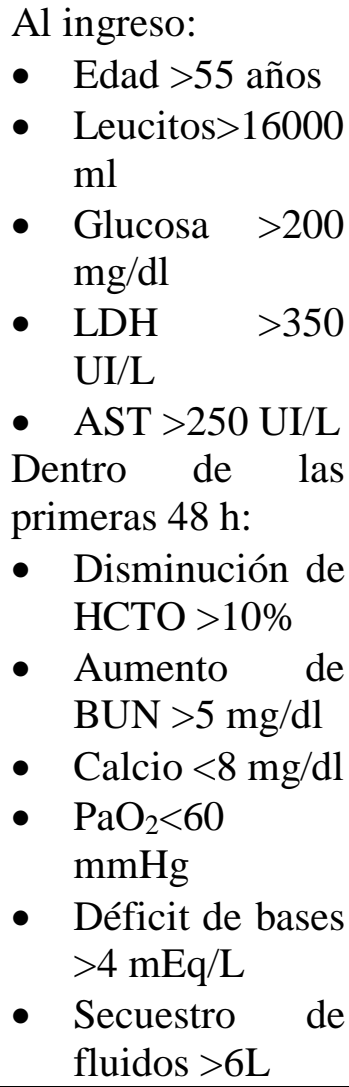 & $\begin{array}{l}\text { BUN } \mathrm{mg} / \mathrm{dl} \text { (urea > }> \\
54 \mathrm{mg} / \mathrm{dl} \text { ) } \\
\text { Alteración del } \\
\text { estado mental } \\
\text { Criterios de } \\
\text { SRIS (>2) } \\
\text { Edad >60 años } \\
\text { Derrame } \\
\text { pleural }\end{array}$ & $\begin{array}{l}\text { A: páncreas } \\
\text { normal } \\
\text { B: páncreas } \\
\text { aumentado de } \\
\text { tamaño } \\
\text { C: rarefacción } \\
\text { der grasa } \\
\text { peripancréatica } \\
\text { D: presencia de } \\
\text { una colección } \\
\text { peripancréatica } \\
\text { E: presencia de } \\
>1 \text { colección } \\
\text { peripancréatica }\end{array}$ & 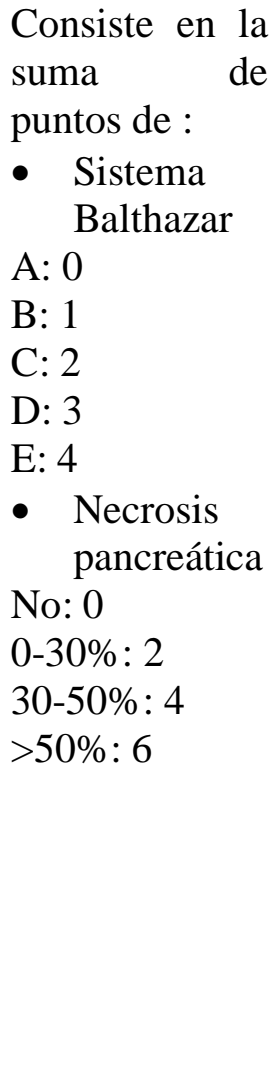 \\
\hline
\end{tabular}

Fuente: Elaboración propia.

La predicción precoz de la gravedad es útil para decidir el ingreso del paciente en una unidad de cuidados intermedios o intensivos. Hay que diferenciar entre gravedad y predicción de gravedad (predecir precozmente si el paciente tendrá un cuadro grave antes de que cumpla los criterios de gravedad). Hay una serie de variables simples asociadas a una peor evolución: edad avanzada, índice de masa corporal mayor de $30 \mathrm{~kg} / \mathrm{m} 2$, presencia de hematocrito mayor de $44 \%$ y/o urea 
mayor de $43 \mathrm{mg} / \mathrm{dL}$ (nitrógeno ureico en sangre > $20 \mathrm{mg} / \mathrm{dL}$ ) en urgencias o que aumentan durante las primeras $24 \mathrm{~h}$, proteína $\mathrm{C}$ reactiva (PCR) mayor de $150 \mathrm{mg} / \mathrm{L}(15 \mathrm{mg} / \mathrm{dL})$ a las $48-72 \mathrm{~h}$ del ingreso y presencia de derrame pleural o infiltrados en la radiografía de tórax. La presencia de criterios SRIS y, sobre todo, su persistencia durante más de 48 h se asocia a peor evolución.

Las complicaciones dentro de la enfermedad pancreática aguda dependen de la gravedad y el pronóstico que ésta tenga. Se puede afirmar que una PA leve (sin complicaciones) tiene un curso muy favorable y se da en el 67\%-69\% de los pacientes; la moderada se asocia a mayor morbilidad (28\%-30\% de los casos), y la grave, a máxima morbilidad y a riesgo de mortalidad (4\%-6\% de los pacientes, con una mortalidad del 30\%-80\%). Las diferentes colecciones pueden dar síntomas al comprimir el tubo digestivo (intolerancia oral) o la vía biliar (ictericia), pueden asociarse a dolor o se pueden infectar. La disrupción del sistema ductal pancreático, generalmente por necrosis pancreática, puede dar lugar a la fuga de jugo pancreático (síndrome del ducto desconectado). La trombosis de las venas esplénica, mesentérica o porta puede asociarse al desarrollo de hipertensión portal izquierda, aunque es raro el sangrado por varices esofagogástricas (20).

Las complicaciones se pueden según un valor pronóstico establecido. Con la clasificación de gravedad de pancreatitis aguda (revisión de la clasificación de Atlanta), tenemos lo siguiente:

- Leve: Ausencia de fallo orgánico, ausencia de complicaciones locales ni sistémicas

- Moderada: Fallo orgánico que se resuelve en 48 h (transitorio) y/o complicaciones locales o sistémicas

- Grave: Fallo orgánico de duración > 48 h (persistente)

\section{Tratamiento}

En las medidas terapéuticas que se tomarán con los pacientes que padezcan de PA pues dependerá de su gravedad. En la PA leve sólo se requiere reposo, ayuno, hidratación endovenosa, reemplazo electrolítico y analgesia. La aspiración por una sonda nasogástrica podría ser útil para disminuir los síntomas, pero no acorta el tiempo de la enfermedad. En las PA moderadas y graves, se necesita manejo en UCI para resucitación adecuada en caso de shock hipovolémico, insuficiencia renal y falla respiratoria. 
El tratamiento de la PAR dependerá de la causa identificada. Si la causa de PAR es debido a drogas, la indicación será suspender la droga responsable y evitar los medicamentos químicamente similares. En caso de una discinecia biliar, la presión elevada de los conductos biliares o pancreáticos se pude disminuir mediante el uso de bloqueadores de los canales de calcio, la esfinterectomía endoscópica o mediante cirugía. El tratamiento de la hipertrigliceridemia es disminuir la ingesta lipídica de la dieta, el ejercicio y el uso de medicamentos. Dentro de los medicamentos, se recomiendan el gemfibrozil, el clofibrato y el ácido nicotínico, habiéndose obtenido mejores resultados con el gemfibrozil. Por otro lado, se han obtenido excelentes resultados en la prevención de PAR idiopática mediante la administración de enzimas pancreáticas en forma profiláctica, ya que disminuiría la frecuencia y severidad de los ataques de PAR idiopática, siendo las enzimas entéricas no recubiertas las que han suprimido efectivamente la secreción del páncreas, dándole reposo y controlando el dolor abdominal. El uso de un adyuvante como los antagonistas de los receptores tipo 2 de histamina o el omeprazol inhibe la secreción ácida y de esta manera limitan la destrucción de las enzimas entéricas recubiertas en el estómago.

Los pacientes con PA presentan un metabolismo similar a los pacientes con sepsis ya que existe un estado hipermetabólico que requerirá de un soporte nutricional adecuado. La recomendación actual en pacientes que no han recibido alimentación por más de 7 a 10 días y que no presenten íleo es el inicio de la nutrición enteral a través de una sonda nasoyeyunal (distal al ángulo de Treitz), que debe controlarse radiológicamente en forma periódica para detectar una eventual migración. La nutrición parenteral total se reserva para pacientes que necesiten un aporte nutricional agresivo, en quienes no se logre un acceso enteral adecuado o que evolucionen de manera desfavorable con la nutrición enteral. El reinicio de la alimentación oral se realizará de acuerdo a los siguientes criterios: ausencia de dolor abdominal, reducción de los niveles de amilasa y lipasa séricos a niveles cercanos a lo normal, ausencia de complicaciones y resolución del íleo con retorno de ruidos hidroaéreos (21).

En pacientes embarazadas el tratamiento es similar que en las pacientes no gestantes, y es fundamentalmente de soporte con colecistectomía electiva después del parto, teniendo como objetivos la prevención y tratamiento de las alteraciones hemodinámicas, supresión de la excreción pancreática, restricción de ingesta de alimentos, tratamiento del dolor, prevención de 
posibles infecciones y tratamiento de las complicaciones, además del estudio de la condición fetal mediante ecografías y monitorización fetal. En las pacientes embarazadas, el tratamiento quirúrgico debe ser reservado para aquellas pacientes en que empeora su condición a pesar del manejo conservador, y puede ser realizado por medio de una colecistectomía la que puede ser segura durante el primer trimestre y después de éste si es necesario. El rol de la colecistectomía laparoscópica permanece controversial debido a reportes disímiles en cuanto a resultados maternos y fetales (22). La colecistectomía raras veces es necesaria durante el embarazo tanto para la colecistitis aguda como para la pancreatitis biliar, pero puede hacerse si es necesario en una forma más segura después del primer trimestre (9).

En otro ámbito con respecto al término del embarazo, aparte de ser un tema controversial, este puede estar indicado en caso de que la paciente no responda al tratamiento conservador o en presencia de complicaciones derivadas de PA. La vía del parto permanece controversial; sin embargo, algunos autores han establecido que la PA durante el embarazo no afecta la indicación de la vía del parto, y en ausencia de contraindicación para parto vaginal, éste es recomendado. Los riesgos fetales están dados principalmente por la prematuridad, la que ocurre en aproximadamente el $60 \%$ de los casos (22).

\section{Conclusiones}

La pancreatitis aguda tiene una incidencia del 0,03 al 0,09\% en las pacientes en embarazadas, es decir que es una entidad poco frecuente siendo las posibles causas de PA en el embarazo son las mismas que en las pacientes no gestantes, entre las que destaca la enfermedad litiásica de la vía biliar como la causa más frecuente; que fue la etiología en el primer episodio de la pancreatitis aguda, de la paciente en el caso clínico descrito.

El tratamiento empleado fue tratamiento médico conservador, evoluciona favorablemente se le da el alta y luego de 3 días presenta dolor abdominal incapacitante, náuseas, vómito de contenido bilioso, exámenes de laboratorio con valores alarmantes de amilasa, lipasa y enzimas hepáticas, llegando al diagnóstico de pancreatitis aguda recidivante secundaria a colelitiasis confirmado por ecografía.

El reporte de caso aporta a la bibliografía y a la práctica clínica, a tener como primera causa de pancreatitis aguda y recidiva de la misma una etiología de origen biliar (microlitiasis y barro 
biliar) siendo los factores predisponentes la disminución de la motilidad de la vesícula biliar y el aumento de la saturación de colesterol que se da en el 10-30\% de las embarazadas. La aplicación de criterios clínicos e imagenológicos contribuyen de forma notable en el pronóstico de la paciente, y el abordaje multidisciplinario de servicios como: ginecología, gastroenterología, medicina interna, unidad de cuidados intensivos y emergencia.

En las pacientes embarazadas, el tratamiento quirúrgico debe ser reservado para aquellas pacientes en que empeora su condición a pesar del manejo conservador, y puede ser realizado por medio de una colecistectomía laparoscópica, posterior a realizar una CPRE. A la paciente se la remite a un hospital de tercer nivel para la realización de una CPRE, y una colecistectomía laparoscópica. Respondiendo favorablemente, sin presentar complicaciones ni aparición de reagudizaciones del proceso inflamatorio del páncreas.

\section{Referencias Bibliográficas}

Alarcón, O. C., Ávila, B., M.L \& Tajmuch V. V. (2008). Pancreatitis Aguda. Revista de Chile de Pediatría. 79(5) Pp. 516-521.

Ampuero, J., Rojas, Feria, M., Gómez, J., Aparcero, R \& Romero, M. (2012). Pancreatitis aguda e infección por Salmonella enteritidis. Revista RAPD online. Julio - Agosto; 35(4). [En línea], Disponible en: https://www.sapd.es/revista/2012/35/4/06.

Argollo, M. P., García, B. C \& Harris, D. P.R. (2007). Pancreatitis aguda recurrente, caso clínico. Revista Chil Pediatr. Abril; 78(2). Pp. 169-175.

Breijo, D.A y Prieto, D.J.A. (2014). Pancreatitis Aguda. IntraMedJournal. Agosto. 3(2).p. 1-14.

Casas, J., Suarez, G., Jiménez, S \&Verona, R. (2010). Pancreatitis aguda necrotizante por cuerpo extraño. Primer caso reportado secundario a un palillo de fósforos. Revista Gastroenterol. Perú. Septiembre. 30(3) Pp. 224-227.

Consejo de Salubridad General. (2010). Diagnóstico y Tratamiento de. Guía de Práctica Clínica para el diagnóstico y tratamiento del hepatoblastoma. Evidencias y recomendaciones. [En línea], Disponible en: http://evaluacion.ssm.gob.mx/pdf/gpc/eyr/SS-287-10.pdf. 
De Madaria, E y Martínez, Sampere, F. (2013). Pancreatitis aguda. Unidad de Patología Pancreática. Unidad de Gastroenterología y Endoscopia Digestiva. Jan.

Domínguez, J.E. (2006). Pancreatitis Aguda. Revista Gastroenterol Hepatol. 29(3) Pp. 77-84.

Farreras, R., Borstnar C, Cardellach. (2016). Medicina Interna. Metabolismo y nutrición. Endocrinología. España. Editorial Elsevier.

Gonzalo, I., Berzosa, J y Díaz, V. (2008). Pancreatitis aguda en el embarazo. Clinic Invest Ginecol Obstet. Febrero; 35(1) Pp. 29-31.

Guachilema, A.P. (2012). Litiasis biliar y su incidencia en pancreatitis aguda, en pacientes de 30 a 65 años de edad en el servicio de cirugía del Hospital Provincial docente Ambato durante el año 2009 a julio 2011. Universidad Técnica de Ambato. 2012 mayo.

Gutiérrez, I., Domínguez, A \& Acevedo, J. (2003). Mecanismos fisiopatogénicos de la pancreatitis aguda. Revista Cirujano General. Vol. 25 Núm. 2. [En línea], Disponible en: http://www.medigraphic.com/pdfs/cirgen/cg-2003/cg032b.pdf.

Harrison, T.R., Kasper, D.L., Hauser, SL., Jameson, J.L., Fauci, A.S \& Longo, D.L. (2016) Principios de Medicina Interna. 19th ed. México, D.F.: McGraw-Hill Interamericana.

Herrera, D.M, Chávez, D.N.C \& Lizardi, D.J. (2003). Pancreatitis aguda y embarazo. Médica sur Sociedad de Médicos A.C. abril-junio. 10(2) Pp. 101-104.

Junquera, R.E., Pereyra, I. (2010). Pancreatitis aguda. Medigraphic: Archivos de Salud Pública. Septiembre - diciembre. 1(1) Pp. 24-30.

Ledesma, P y Arias, J. (2009). Pancreatitis aguda. Medicina Interna de México. 25(4) Pp. 285294.

Pandol, S. (2005). Pancreatitis aguda: experiencia de cinco años en el Hospital General de México. Revista Gac. Méd. Méx. Vol. 141 N.2. Pp. 538-543.

Pellegrini, D., Pankl, S., Finn, B.C., Bruetman, J.E, Zubiaurre. I \& Young, P. (2009). Pancreatitis aguda. Análisis de 97 pacientes. Medicina (B. Aires). Medicina (Buenos Aires). 69(2), 239-245. 
[En línea], Disponible en: http://www.scielo.org.ar/scielo.php?script=sci_arttext\&pid=S0025$76802009000300004 \& \operatorname{lng}=$ es\&tlng=es.

Pérez, M. (2002). Pancreatitis Aguda: Valoración de la Gravedad. Revista GH CONTINUADA. Julio-Agosto; 1(5).Pp. 221-225.

Vaillant, R.S., Rodríguez, A.A \& López, G.A. (2012). Pancreatitis Aguda. [En línea], Disponible en: http://files.sld.cu/cirured/files/2012/03/pancreatitis.pdf.

Vergara, J.M y Buforn, A. (2002).Rodríguez Serrano C. Pancreatitis Aguda. Pp. 1-13. [En línea], Disponible en: http://www.medynet.com/usuarios/jraguilar/Manual\%20de\%20urgencias\%20y\%20Emergencias/ pancreag.pdf. 